\title{
A Role for Conditioned Ventral Tegmental Glutamate Release in Cocaine Seeking
}

\author{
Zhi-Bing You, Bin Wang, Dawnya Zitzman, Soraya Azari, and Roy A. Wise \\ Behavioral Neuroscience Branch, Intramural Research Program, National Institute on Drug Abuse, National Institutes of Health, Baltimore, Maryland \\ 21224
}

Initiation of cocaine self-administration in rats was associated with release of glutamate in the ventral tegmental area (VTA). The glutamate release was transient, despite continued cocaine intake. Similar glutamate release was seen in rats earning, for the first time, unexpected saline rather than expected cocaine. VTA glutamate release was not seen in similarly trained rats earning saline instead of cocaine for the 13th time. VTA glutamate release was also seen in similarly trained rats that received yoked rather than earned cocaine injections on test day. VTA glutamate release was not seen in a group of rats that had never earned cocaine but had received yoked injections during the training period. Glutamate release was also not seen in a group of rats that received yoked injections but had no previous experience with cocaine. VTA GABA levels did not fluctuate during any aspect of cocaine seeking. Blockade of VTA glutamate receptors appeared to attenuate the rewarding effects of intravenous cocaine injections and blocked almost completely the conditioned responding normally seen during extinction trials. These findings indicate that VTA glutamate release is a conditioned response dependent on an associative process and is not a simple consequence of previous cocaine exposure. The findings implicate glutamate as at least one of the sources of VTA signals from reward-associated environmental stimuli.

Key words: cocaine; extinction; GABA; glutamate; self-administration; ventral tegmental area

\section{Introduction}

A variety of natural and laboratory rewards are habit-forming mainly because they elevate extracellular dopamine levels (Wise, 2004). Under conditions of dopamine receptor blockade, food that is normally rewarding fails to establish new lever-pressing habits (Wise and Schwartz, 1981), and such rewards as food (Wise et al., 1978), brain stimulation (Fouriezos and Wise, 1976), and the psychomotor stimulants amphetamine (Yokel and Wise, 1975) and cocaine (de Wit and Wise, 1977) fail to maintain habitual responding in trained animals. Whereas natural rewards like food and sexual interaction can double dopamine levels (Hernandez and Hoebel, 1988), self-administered cocaine, heroin, and amphetamine can elevate dopamine levels fivefold or more (Hurd et al., 1989; Pettit and Justice, 1989; Wise et al., 1995a,b; Ranaldi et al., 1999).

Even the rewarding effects of cocaine, involving cocaine's action at dopaminergic terminals in nucleus accumbens, olfactory tubercle, and medial prefrontal cortex (Roberts et al., 1977; Goeders and Smith, 1983; Carlezon and Wise, 1996; Ikemoto, 2003), depend on afferent input to midbrain [ventral tegmental area (VTA)] dopaminergic cell bodies. Cocaine elevates dopamine

Received Dec. 1, 2006; revised Aug. 16, 2007; accepted Aug. 17, 2007.

This work was supported by the Intramural Research Program of the National Institutes of Health (National Institute on Drug Abuse).

Correspondence should be addressed to Zhi-Bing You, Intramural Research Program, National Institute on Drug Abuse, 5500 Nathan Shock Drive, Baltimore, MD 21224. E-mail: zyou@intra.nida.nih.gov.

D. Zitzman's present address: Psychology Department, Tufts University, Medford, MA 02155.

S. Azari's present address: University of Pennsylvania Medical School, Philadelphia, PA 19104.

D0I:10.1523/JNEUROSCI.2967-07.2007

Copyright $\odot 2007$ Society for Neuroscience ～0270-6474/07/2710546-10\$15.00/0 levels by blocking the monoamine transporters by which extracellular dopamine is cleared (Heikkila et al., 1975; Morón et al., 2002). For cocaine to sustain elevated dopamine levels, it is necessary for dopaminergic cells to remain activated, despite autoreceptor inhibition, either by increased excitatory or decreased inhibitory input. The primary excitatory and inhibitory inputs thought to provide such control are glutamate and GABA, respectively (Grace and Bunney, 1979). Glutamate input to the VTA has been implicated in several aspects of cocaine seeking (Harris and Aston-Jones, 2003; Sun et al., 2005; Wang et al., 2005). We use the term "cocaine seeking," here and throughout this paper, as descriptive shorthand to indicate initiation or maintenance of an instrumental response habit that has been established by response-contingent application of cocaine in the animal's reinforcement history. The term is used to indicate that the behavior depends primarily on the animal's reinforcement history but can persist for long periods without continued reinforcement.

Although the rewarding effects of cocaine depend on activation of the dopamine system, recent electrophysiological studies have suggested that the system, like the act of lever pressing, is often more directly activated by reward-predictive environmental stimuli than by the receipt of a primary reward itself (Schultz, 1997). Reward-predictive stimuli cause brief burst firing of dopamine neurons, presumably by increasing excitatory input, but the afferent pathways responsible for this input remain to be identified (Dommett et al., 2005). It is known that experimenteradministered cocaine induces VTA glutamate release in cocaineexperienced but not cocaine-naive rats; this release has been at- 
tributed to cocaine sensitization rather than to cocainepredictive cues or cocaine expectation (Kalivas and Duffy, 1998).

In the present study, we used brain microdialysis to characterize VTA glutamate and GABA fluctuations in rats selfadministering or passively receiving cocaine after various kinds of cocaine experience and in the presence of various cocainepredictive cues. We then confirmed the importance of VTA glutamate for cocaine seeking by blocking the effects of ionotropic VTA glutamate receptors during cocaine self-administration and extinction responding.

\section{Materials and Methods}

Two sets of studies were done. In the first set of studies, microdialysis samples were taken from rats self-administering intravenous cocaine, undergoing extinction, or receiving "yoked" cocaine injections. These groups differed as to their training or testing conditions as described below. In the second set of studies, rats self-administering intravenous cocaine were challenged with reverse dialysis administration of glutamate antagonists. For both the microdialysis studies and the reverse dialysis studies, the dialysis probes were implanted in the VTA, just dorsal to the VTA, or just lateral to the VTA.

\section{Subjects}

A total of 194 350-400 g male Long-Evans rats (Charles River, Raleigh, NC) were used. They were housed individually under a reverse light/dark cycle (light on from 8:00 P.M. to 8:00 A.M.) with ad libitum access to food and water. They were allowed to acclimate to the new environment for at least $7 \mathrm{~d}$ before surgery. The experimental procedures followed the Guide for the Care and Use of Laboratory Animals published by National Institutes of Health (1996) and were approved by the local Animal Care and Use Committee.

\section{Surgery}

Each rat was implanted with unilateral or bilateral guide cannulas for microdialysis probes and with an indwelling jugular catheter for intravenous drug injections. The animal was anesthetized with a mixture of pentobarbital and chloral hydrate (30 and $140 \mathrm{mg} / \mathrm{kg}$, i.p., respectively) and placed in a stereotaxic frame with the incisor bar set $5.0 \mathrm{~mm}$ dorsal to the intra-aural line.

Intracranial cannulation. For experiments involving measurement of VTA transmitter release, unilateral guide cannulas (CMA/Microdialysis, North Chelmsford, MA) were implanted above the left VTA, at an angle of $12^{\circ}$ toward the midline (to avoid puncture of the sagittal sinus). The cannulas were zeroed at bregma, moved $2.2 \mathrm{~mm}$ lateral and $5.6 \mathrm{~mm}$ posterior, and rezeroed at the surface of the skull; they were then lowered $6.7 \mathrm{~mm}$ along their angled path and cemented in place. For "dorsal control" rats, the cannulas were lowered only $5 \mathrm{~mm}$ along the angled path. For "lateral control" rats, the cannulas were implanted $1.1 \mathrm{~mm}$ further lateral, targeting the substantia nigra (SN) adjacent to the VTA. For experiments involving perfusions of glutamate antagonists, bilateral cannulas were implanted. Once the cannulas were positioned, they were anchored to four skull screws with dental cement.

Intravenous catheterization. After fixation of the guide cannulas, the rats were implanted with chronically indwelling intravenous catheters. A small incision was made to the right of the midline of the neck, and the external jugular vein was isolated. A length of SILASTIC tubing (Dow Corning, Midland, MI) was inserted to just reach the right atrium and was secured to the vein with a silk suture. The other end of the catheter was fed subcutaneously to exit near the back of the skull, where it was connected to a capped 22 gauge stainless-steel cannula fitting (Plastics One, Roanoke, VA). The fitting was cemented to the guide cannula assembly, and the wound was sutured.

After surgery, each rat was given $0.25 \mathrm{ml}$ of subcutaneous enrofloxacin (Baytril; 2.27\%); these injections were continued daily for $3 \mathrm{~d}$ as precaution against infection. The animals were allowed to recover for at least $5 \mathrm{~d}$ before the behavioral training started. The catheters were flushed daily with $0.05 \mathrm{ml}$ of gentamicin $(4 \mathrm{mg} / \mathrm{ml}$ dissolved in sterile saline) and 0.05 $\mathrm{ml}$ of heparin $(30 \mathrm{U} / \mathrm{ml}$ dissolved in sterile saline) until the experimental testing began.

\section{Microdialysis studies}

Each experiment involved a $12 \mathrm{~d}$ training phase and a subsequent testing phase; one group had a $12 \mathrm{~d}$ extinction phase between training and testing. The rats were assigned to experimental groups of size 7 after successfully completing their assigned training phase. Final sample sizes varied from 5 to 7 and are individually noted in Results as the findings from each group are described.

Training phase. After recovery from surgery, most animals were given normal self-administration "training" $6 \mathrm{~d}$ per week in $4 \mathrm{~h}$ sessions. Each rat's catheter was connected daily by polyethylene tubing, through a feed-through swivel that allowed freedom of movement, to a microprocessor-controlled syringe pump (Razel Scientific instruments, Stamford, CT). The rat was then placed in a dimly (red) lit operant chamber equipped with two levers, one fixed and one retractable (Med Associates, Georgia, VT), protruding from the cage wall, $9 \mathrm{~cm}$ above the floor. Training sessions began with the insertion of the retractable lever into the chamber and illumination of the house light that remained on throughout the session. Depression of the retractable lever (designated the "active" lever) caused delivery of intravenous cocaine $\left(1 \mathrm{mg} \cdot \mathrm{kg}^{-1}\right.$. injection ${ }^{-1}$ ) or saline, in a volume of $0.13 \mathrm{ml}$, delivered over $4.5 \mathrm{~s}$ on a fixed ratio-1 schedule of reinforcement. A white cue light above the lever was illuminated with the onset of the infusion and remained illuminated for $20 \mathrm{~s}$ during a "time out" in which further lever pressing was ineffective. Depressions of the fixed lever (designated the "inactive" lever) were recorded but had no consequences for the animal. No priming injections or response shaping were given. One of these groups was trained in a different room, with contextual cues that differed from those where they were tested. For this group, the training room was larger and had a different pattern of noise and overhead lights, and the chamber was equipped with a dim yellow house light rather than the dim red house light of the test chamber. These animals were designated the "novelroom" group; after training, they were tested in the same chambers as the other groups.

Two groups, to be eventually tested with yoked rather than earned injections, were not given the opportunity to self-administer cocaine during the training phase. One group, designated the cocaine-naive group, was given the opportunity to self-administer saline rather than cocaine; their training conditions were normal except that their syringes contained physiological saline rather than cocaine. Thus this group received the same handling and the same experience with the apparatus and daily procedure but had no exposure to cocaine before testing in the yoked injection experiments. The second group, designated the yoked history group, was given unearned rather than earned cocaine injections throughout the training period; the timing of each animal's injections was controlled by a yoked partner that earned injections for both animals. Here, illumination of the house light accompanied the onset of the yoked injection period, and the cue light was illuminated for $20 \mathrm{~s}$ with the onset of each injection. Lever presses were recorded but had no consequence for the animals in this group. Thus this group received the same handling and the same pharmacological exposure to cocaine as did the normally trained animals; however, these animals were never given response-contingent cocaine injections.

Testing phase. Microdialysis samples were taken from three groups of normally trained animals; one group had its dialysis probes in the VTA, one had its probes in the $\mathrm{SN}$, and one group had its probes in the dorsal control (DC) position. These animals were tested under cocaine selfadministration conditions identical to those of training.

Microdialysis samples were also taken from four groups of animals tested under extinction conditions. Three groups differed by probe placement, with probes in the VTA, SN, and DC positions. These animals were tested under conditions identical to those of cocaine selfadministration training, except that saline was substituted for cocaine in the animals' syringes. The fourth group, with VTA probes, was given $12 \mathrm{~d}$ of extinction training after their $12 \mathrm{~d}$ of cocaine self-administration training. Microdialysis samples were taken from these animals on their 13th day of extinction experience. Each saline injection during extinction test- 
ing, like each cocaine injection in the previous training sessions, was accompanied by illumination of the cue light above the lever.

Next, microdialysis samples were taken from five groups of animals that received yoked rather than earned cocaine injections. Here, the rate of injection was not determined by the animal being tested, but rather by a yoked partner that earned the injections under the training conditions described earlier. Illumination of the cue light over the active lever (or its empty slot) accompanied each injection. The five groups differed in their treatment during the normal training period. Three of these groups were trained normally as described above. The first group was designated "lever withheld" and was tested with the retractable lever in the retracted position. These animals simply received, for the first time, yoked injections in the absence of their response lever. The second group was designated lever inserted and was tested normally except that cocaine injections were yoked rather than earned. That is, for this group the retractable lever was inserted as usual but was disconnected from the infusion pump. Thus lever pressing did not, for this test in this group, result in cocaine injections; rather, yoked cocaine injections were given under the control of the yoked partner. The third group, designated novel room, was also tested in the lever-inserted condition, but differed from the other groups in that it was trained in a room with different contextual cues (lighting and lab noise).

The two remaining groups receiving yoked injections had the unusual training conditions mentioned earlier. One group, designated cocaine naive, was exposed to intravenous saline but not cocaine reward in the training phase. The other group, designated yoked history, was exposed only to yoked injections throughout the training phase; thus, for these animals, dialysis samples were taken on the 13th day of yoked injections. These groups, like those with a self-administration history, simply received unsignaled yoked injections during their microdialysis test day.

Finally, to determine whether the observed glutamate release was dependent on neuronal impulse flow, another group of animals was tested during infusion with tetrodotoxin (TTX), a sodium channel blocker. TTX infusion was initially given to four animals during cocaine selfadministration, but each animal simply failed to respond for cocaine during TTX infusions, and there were no glutamate peaks to block in this condition. Because there was no cocaine administered under these conditions, we instead challenged the glutamate response to yoked cocaine injections. In this case, the animals were tested in the lever-withheld condition, the condition that previously gave the strongest glutamate release and thus the strongest opportunity to detect effects of challenge.

\section{Microdialysis procedure}

Microdialysis during cocaine self-administration, extinction, and yoked cocaine injections. Microdialysis probes ( $2 \mathrm{~mm}$ active membrane) were purchased from CMA/Microdialysis (CMA/12; CMA/Microdialysis). The length of active membrane was $2 \mathrm{~mm}$; when the probes were inserted, only the active membrane protruded beyond the length of the implanted guide cannula. After the last day of self-administration training, the dummy probe on each animal's guide cannula was removed, and a microdialysis probe was inserted, fixed in position, and connected to the microdialysis pump (CMA/100). The animal was kept in selfadministration box overnight with food and water available ad libitum, and the probe was perfused with artificial CSF (aCSF) at a flow rate of 0.4 $\mu \mathrm{l} / \mathrm{min}$. The following morning, the flow rate was increased to $2 \mu \mathrm{l} / \mathrm{min}$ for the remainder of the experiment. The composition of the aCSF was $\mathrm{NaCl}$ (148 mM), $\mathrm{KCl}$ (2.7 mM), $\mathrm{CaCl}_{2}$ (1.2 mM), and $\mathrm{MgCl}_{2}$ (0.85 mM). Four 10 min baseline samples were collected, starting $1 \mathrm{~h}$ after the increase in flow rate, and then the $4 \mathrm{~h}$ testing sessions began. Ten minute samples were collected throughout and were split for analysis of glutamate $(10 \mu \mathrm{l})$ and GABA $(10 \mu \mathrm{l})$.

Microdialysis with TTX perfusion. In one group of trained rats $(n=4)$, TTX $(0.1 \mu \mathrm{M})$ was added to the perfusion medium $30 \mathrm{~min}$ before the collection of the baseline samples, and the rats were then allowed to self-administer cocaine. In a second group of trained rats, yoked injections were given in the lever-withheld condition. Again, TTX $(0.1 \mu \mathrm{M})$ was added to the perfusion medium $30 \mathrm{~min}$ before the collection of the baseline samples and remained in the medium throughout the period of yoked injections.
Biochemical analyses

Glutamate determination. HPLC was used for determination of glutamate; our system comprises a model 582 HPLC pump (ESA, Chelmsford, MA), a CMA/260 degasser, a CMA/200 refrigerated microsampler, a phase II ODS column $(3 \mu \mathrm{m}$ particle size, $3.2 \times 100 \mathrm{~mm}$; Bioanalytical Systems, West Lafayette, IN), a CMA/280 fluorescence detector, and an ESA model 501 data station (ESA). The CMA/280 is a fixed-wavelength fluorescence detector operating at a maximal excitation of $330-365 \mathrm{~nm}$ and emission of $440-530 \mathrm{~nm}$. The precolumn derivatization of glutamate was performed with an $o$-phthalaldehyde/mercaptoethanol reagent (0.4 M borate, $0.04 \mathrm{~m}$ phthalaldehyde, and $0.4 \mathrm{~m} 2$-mercaptoethanol, $\mathrm{pH}$ 10.4). Briefly, $10 \mu \mathrm{l}$ of the reagent was added to and mixed with the samples by the microsampler. After a $60 \mathrm{~s}$ reaction period at $6^{\circ} \mathrm{C}$ in the microsampler, $20 \mu \mathrm{l}$ of the mixture was injected onto the column. The elution of glutamate was achieved with a mobile phase consisting of 0.15 M sodium acetate, $10 \%$ methanol, and $1.5 \%$ tetrahydrofuran at a flow rate of $0.6 \mathrm{ml} / \mathrm{min}$. After the appearance of the glutamate peak on the chromatogram, an injection of $20 \mu \mathrm{l}$ of $100 \%$ methanol was made by the microsampler before the end of the chromatogram to accelerate the elution of the residuals on the column. The detection limit was 0.2 $\mathrm{pmol} /$ injection.

GABA determination. The HPLC system for determination of GABA was similar to that for determination of glutamate, except that a microbore column ( $3 \mu \mathrm{m}$ particle size, $150 \times 1 \mathrm{~mm}$, C-18; Bioanalytical Systems) was used. The precolumn derivatization of GABA was performed by adding $1 \mu \mathrm{l}$ of the reagent into the samples. The elution of GABA was achieved with an acetate mobile phase $(0.1 \mathrm{M}$ sodium acetate, $20 \%$ acetonitrile, and 2.5\% 1-propanol, $\mathrm{pH}$ adjusted at 5.4 with phosphoric acid) at a flow rate of $70 \mu \mathrm{l} / \mathrm{min}$. The column wash procedure was done by injecting $10 \mu \mathrm{l}$ of acetonitrile before the end of the analysis. The detection limit with the micro bore system was $20 \mathrm{fmol} /$ injection.

\section{Challenge with glutamate antagonists}

The microdialysis studies described above revealed fluctuations in VTA levels during cocaine self-administration and the first day of extinction testing. To determine the importance of VTA glutamate fluctuations for the behavior of the animals, we tested the effects of bilateral VTA perfusion of various glutamate antagonists. Initially, we tested the effects of kynurenic acid (Kyn), an ionotropic glutamate receptor antagonist, on cocaine self-administration and extinction responding. Because Kyn is a nonselective ionotropic glutamate antagonist and can also act at $\alpha-7$ nicotinic receptors (Hilmas et al., 2001) that can modulate glutamate release in the VTA (Jones and Wonnacott, 2004), we subsequently used more-selective glutamate antagonists to challenge the glutamate contribution to responding. Here we tested the effects of DL-2-amino-5phosphonopentanoic acid (AP-5; a selective NMDA-type glutamate antagonist) and 6-cyano-7-nitroquinoxaline-2,3-dione (CNQX; a selective AMPA/kainite-type glutamate antagonist). For these tests, antagonists were given bilaterally by reverse dialysis during extinction testing. A three-channel swivel was used to allow the animal ad libitum movement; one channel was used for intravenous injections, and two were used for reverse dialysis.

Kyn during self-administration. Six normally trained groups were tested with VTA infusions of the broad-spectrum ionotropic glutamate antagonist kynurenic acid. Two of these groups were tested with Kyn (1 $\mathrm{mm}$ ) or vehicle (aCSF) added to the perfusion medium $1 \mathrm{~h}$ before the levers were introduced into the chambers to initiate the "loading phase" of self-administration sessions. The remaining four groups were tested with $\operatorname{Kyn}(0,0.1,1.0$, or $5.0 \mathrm{~mm})$ added to the perfusion medium $1 \mathrm{~h}$ after the levers were introduced, during the "maintenance phase" of cocaine self-administration. Except for the reverse dialysis, these sessions were identical to the normal training sessions.

Kyn during extinction. Five groups of rats were tested to determine the effects of Kyn on lever pressing under extinction conditions. Three groups were tested with VTA Kyn $(0.1$ or $1 \mathrm{~mm})$ or aCSF added to the microdialysis perfusion medium. One group was tested with Kyn infusion $(0.1 \mathrm{~mm})$ through SN probes and one with the same dose through DC probes. Here, Kyn was added to the perfusion medium during the baseline period, $60 \mathrm{~min}$ before insertion of the retractable lever and ini- 
tiation of the extinction session. Except for the reverse dialysis, these sessions were identical to the extinction sessions described above.

$A P-5$ and $C N Q X$ in extinction. To determine the relative contributions of the two major subtypes of ionotropic glutamate receptor antagonists, four groups of rats $(n=7$ each) were treated with AP-5 (0.1 mM), CNQX (0.1 mM), AP-5 plus CNQX ( $0.1 \mathrm{~mm}$ in each case), or aCSF under extinction conditions. In each case the antagonists were introduced to the dialysis medium $60 \mathrm{~min}$ before insertion of the retractable lever and initiation of extinction sessions. Except for the reverse dialysis, these sessions were identical to the extinction sessions described above.

\section{Drugs}

Cocaine hydrochloride and the anesthetic mixture were obtained from the Pharmacy Department of the National Institute on Drug Abuse, Intramural Research Program. Kyn, AP-5, CNQX, and TTX were purchased from Sigma-Aldrich (St. Louis, MO). Kyn was first dissolved with a small aliquot of $5 \mathrm{~N} \mathrm{NaOH}$ and diluted with aCSF to the final concentrations. AP-5, CNQX, and TTX were dissolved directly in aCSF. Each drug solution was adjusted to $\mathrm{pH} 7.4$ before use.

\section{Histology}

After the completion of the microdialysis experiment, the rats were decapitated under deep anesthesia, and the brains were removed and fixed in $10 \%$ formalin. Fifty micrometer coronal sections were sliced with a cryostat. The probe location was examined under microscope at low magnification in wet tissue that allowed differentiation of fiber tracts and cell groups. Probe locations were reconstructed on drawings from the atlas of Paxinos and Watson (1998).

\section{Statistics}

The levels of the assayed amino acids were expressed as the concentrations found in the perfusates (means and SEM). Basal values refer to those obtained before the drug infusion or lever press was initialized. When data were expressed as percentages of basal levels, the mean concentration of the three samples preceding the drug self-administration session or the behavior testing was defined as $100 \%$. The effects of the drug or behavioral performance on the transmitter release and the effects of the drug on lever presses were analyzed with one-way or two-way ANOVA with repeated measures over time. Fisher's PLSD test was used for comparisons to pretreatment baseline or between the experimental groups. A level of $p<0.05$ for a two-tailed test was considered critical for statistical significance. Curve fitting was done by SigmaPlot graphics software (Systat Software, Point Richmond, CA). Pearson product-moment correlations were calculated between responses per session and glutamate elevations (first two samples) for animals in the selfadministration, extinction, and yoked injection conditions.

\section{Results}

Glutamate and GABA release associated with initiation of cocaine self-administration

Glutamate levels in the VTA increased to $170 \%$ of baseline in the first half-hour of self-administration sessions (Fig. $1 B$ ). Two-way ANOVA confirmed a significant group $\times$ time interaction (Fig. $1 B)\left(F_{(52,390)}=1.52 ; p<0.05\right)$, which resulted from the significant peak in the VTA (Fisher's PLSD test, $p<0.05$ ); there were no significant changes in or differences between the SN or DC samples. VTA glutamate levels returned to normal within $1 \mathrm{~h}$, despite the fact that lever pressing and the resulting cocaine injections continued at a steady pace for the full $4 \mathrm{~h}$ of drug availability (Fig. $1 D$ ). Throughout this period, dopamine levels in nucleus accumbens typically remain elevated to $300-400 \%$ of baseline (Pettit and Justice, 1989; Wise et al., 1995a). Differences in the glutamate peaks between animals were not correlated with differences in the rates of lever pressing $(r=0.29 ; p>.25)$. Baseline levels of glutamate were $1.1 \pm 0.2,1.3 \pm 0.2$, and $1.2 \pm 0.4 \mu \mathrm{M}$, respectively, for animals with microdialysis probes in the VTA $(n=7)$, $\mathrm{SN}(n=6)$, or DC $(n=5)$; there were no significant differences in baseline glutamate levels between groups.
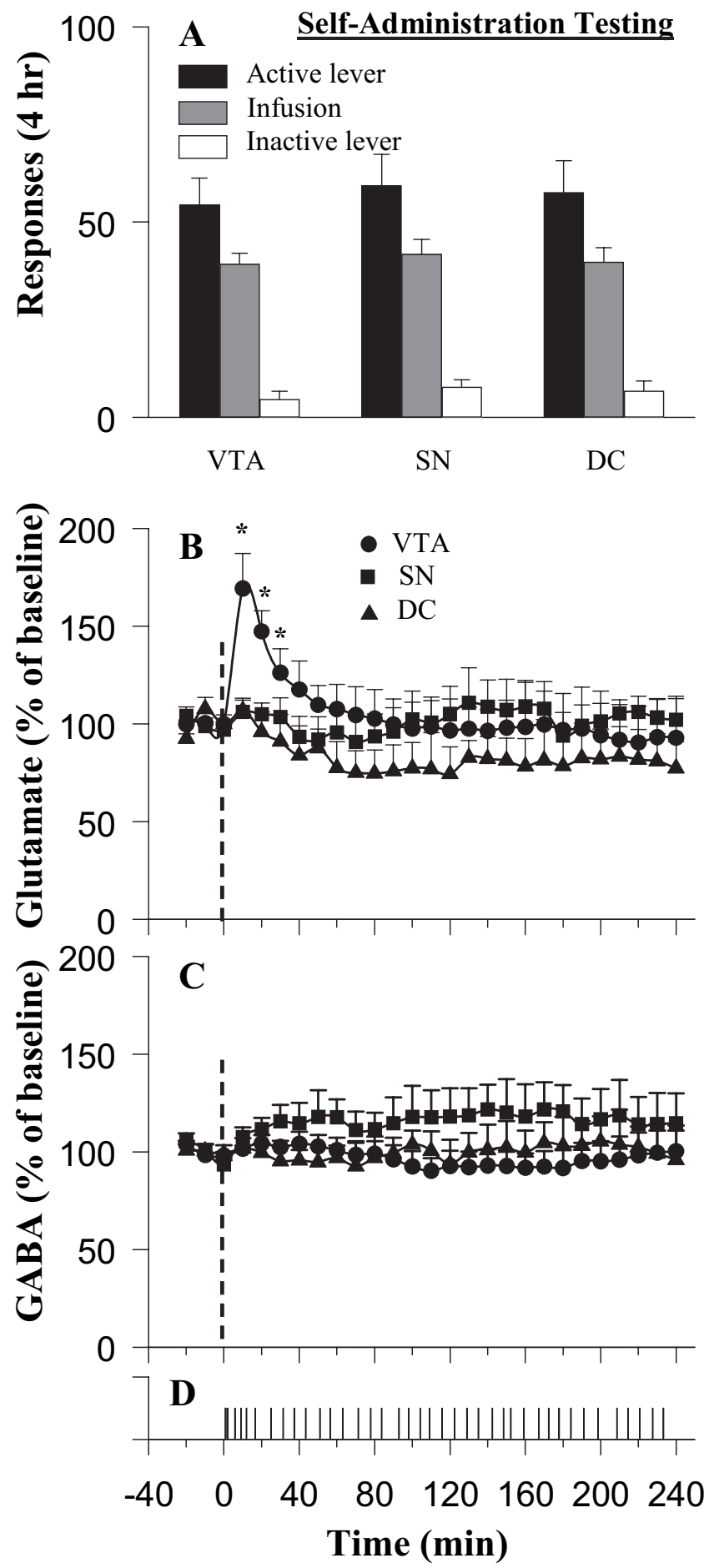

Figure 1. $\quad A-C$, Behavioral responses $(A)$ and fluctuations in mean levels of glutamate $(B)$ and $G A B A(C)$ during intravenous cocaine self-administration. $D$, A typical response record for a rat with microdialysis probe implanted in the VTA. Glutamate levels in the VTA but not in the region dorsal (DC) or lateral (SN) to the VTA were significantly increased after the initiation of the self-administration session. The dotted vertical lines at time $=0$ indicate the start of the self-administration session. The asterisk $\left(^{*}\right)$ indicates significant $(p<0.05)$ difference from baseline levels.

GABA levels did not differ between groups and did not fluctuate with time. Two-way ANOVA revealed no significant effect of group or time and no group $\times$ time interaction $\left(F_{(26,156)}=\right.$ $1.12 ; p=0.33$. Basal GABA levels were $22.7 \pm 5.2 \mathrm{~nm}$ for the 
VTA, $25.4 \pm 5.4 \mathrm{~nm}$ for the SN, and $17.2 \pm 2.1 \mathrm{~nm}$ for the DC groups.

\section{Glutamate and GABA release associated with responding in extinction.}

When trained animals were first tested in extinction conditions, they lever pressed avidly (Fig. 2A). Response rates in the first extinction session did not differ among the VTA $(n=7), \mathrm{SN}(n=$ $6)$, and DC $(n=6)$ groups (Fig. $2 A$, session 1$)$. The animals responded strongly and preferentially on the active lever in the initial portion of the session (Fig. 2D); rats in this salinesubstitution condition moved away from the lever after the first hour and tended to rest or sleep for the remainder of the session. By the 13th extinction session, the animals responded minimally (Fig. 2A,E).

Despite the absence of cocaine reinforcement, VTA glutamate levels again peaked at $170 \%$ of baseline in the first trial of extinction testing (Fig. $2 \mathrm{~B}$ ). Two-way ANOVA among the four groups revealed a significant time $\times$ group interaction $\left(F_{(42,294)}=2.01\right.$; $p<0.01)$; Fisher's PLSD test confirmed that VTA glutamate was elevated on the first but not on the 13th day of extinction and that SN and DC glutamate did not vary significantly. In this case, the elevations of VTA glutamate level were strongly correlated with the rates of responding on the active lever $(r=0.86 ; p<0.01)$. Here, glutamate levels returned to normal more quickly and to somewhat lower levels than they had when rats received their expected cocaine. Unlike the case when animals continued earning cocaine, the decline of the glutamate signal in extinction was accompanied by a decline in lever pressing (Fig. 2D).

Two-way ANOVA revealed no significant interaction of GABA as a function of time $\times \operatorname{group}\left(F_{(42,294)}=0.66 ; p=0.95\right)$ (Fig. 2C).

\section{Glutamate and GABA release associated with yoked cocaine injections}

Lever pressing varied significantly in the yoked injection condition. One-way ANOVA revealed a main effect of groups on active lever responding among the four groups with access to the active lever $\left(F_{(3,24)}=10.26 ; p<0.001\right)$; Fisher's PLSD test confirmed that responding in the two trained groups with access to their levers [lever inserted $(n=7)$ and novel room $(n=7)$ ] was significantly higher than the minimal responding for the two groups that had access to the active lever but had no history of cocainereinforced lever pressing [yoked history $(n=7)$ and cocaine naive $(n=7)]$. Because the active lever was not inserted for the lever-withheld group $(n=7)$, there was no measure of active lever responding in this group. These animals were observed to approach the wall slot in which the lever was inserted during training days, scrabbling at the slot with their forepaws. For responding on the inactive lever, one-way ANOVA again revealed a main effect of group $\left(F_{(4,30)}=3.35 ; p<0.05\right)$; in this case, Fisher's PLSD test confirmed that the animals without access to the active lever (lever-withheld group) differed significantly from the four groups with access to the active lever (Fig. $3 A)(p<0.01)$. Basal glutamate level in cocaine self-administration-trained (lever withheld, lever inserted, and novel room) animals was $1.4 \pm$ $0.2 \mu \mathrm{M}$; basal level in the yoked-trained (yoked history) animals was $1.3 \pm 0.3 \mu \mathrm{M}$; and basal level in the cocaine-naive group was $1.0 \pm 0.3 \mu \mathrm{M}$.

VTA glutamate elevations varied greatly across the five groups that received yoked injections (Fig. 3B). An overall two-way ANOVA with repeated measures for the five groups confirmed a significant main effect of time $\left(F_{(26,806)}=10.28 ; p<0.001\right)$ and a significant time $\times$ group interaction $\left(F_{(130,806)}=1.55 ; p<\right.$ $0.001)$. Fisher's PLSD tests confirmed that only the glutamate peaks for the lever-withheld $(n=6)$, lever-inserted, and novelroom conditions were significantly elevated (Fig. $3 B$ ). Whereas the glutamate elevations in the lever-inserted and novel-room conditions were similar to those seen in cocaine selfadministration conditions (compare with Fig. $1 B$ ), the elevations in the lever-withheld group were significantly higher and longer lasting.

Glutamate peak elevations were correlated with response rates for the two groups that were previously trained to self-administer cocaine and were subsequently tested with yoked injections. These were the lever-inserted $(r=0.83 ; p<0.01)$ and the novelroom $(r=0.92 ; p<0.01)$ groups. The other trained group tested with yoked injections was the lever-withheld group, the group that did not have access to the active lever. These animals were seen to approach and scrabble at the wall slot in which the active lever was normally inserted, and these animals pressed the alternative, inactive lever (Fig. 3A). However, responding on the inactive lever was not correlated with the peak glutamate elevations in this group $(r=0.19 ; p>0.35)$.

VTA GABA levels did not fluctuate significantly in any of the groups with previous cocaine experience, but were slowly elevated in the cocaine-naive group (Fig. $3 C$ ). Two-way ANOVA with repeated measures for the five groups confirmed a significant main effect of time $\left(F_{(26,806)}=6.02 ; p<0.001\right)$ and significant time $\times$ group interaction $\left(F_{(130,806)}=1.72 ; p<0.001\right)$. Fisher's PLSD tests confirmed that only the GABA peak for the cocaine-naive group was significantly elevated. Basal GABA level in cocaine self-administration-trained (lever withheld, lever inserted, and novel room) animals was $23.2 \pm 2.7 \mu \mathrm{M}$; basal level in the yoked-trained (yoked history) animals was $24.4 \pm 3.5 \mu \mathrm{M}$; and basal level in the cocaine-naive group was $19.6 \pm 3.3 \mu \mathrm{M}$.

The four trained animals given the opportunity to selfadminister cocaine during VTA perfusion with TTX failed to lever press and thus received no cocaine. When yoked cocaine injections were given to animals in the lever-withheld condition $(n=7)$, TTX infusion prevented the glutamate increase that was seen in untreated animals (Fig. 4A). Two-way ANOVA with repeated measures confirmed a time $\times$ group interaction $\left(F_{(14,154)}\right.$ $=7.32 ; p<0.001)$; Fisher's PLSD test confirmed no significant increase in glutamate levels in TTX-treated animals. There was no significant effect of TTX on GABA levels (Fig. 4B). Basal glutamate levels in control and TTX treated group were $1.2 \pm 0.3$ and $1.6 \pm 0.4 \mu \mathrm{M}$, respectively; basal GABA levels were $26.3 \pm 4.9$ and $22.2 \pm 3.8 \mathrm{nM}$, respectively.

\section{Effects of VTA glutamate receptor blockade on cocaine self-administration}

Rats given $1.0 \mathrm{~mm}$ Kyn through the VTA dialysis probe for $60 \mathrm{~min}$ before and during cocaine self-administration testing accelerated their responding for cocaine (Fig. $5 A$ ) (treatment $\times$ time interaction, $\left.F_{(23,276)}=14.32 ; p<0.001\right)$ and, consequently, their hourly drug intake. Similarly, when Kyn ( 1.0 and 5.0 but not 0.1 $\mathrm{mm}$ ) was introduced $1 \mathrm{~h}$ after initiation of cocaine selfadministration, rats increased their responding (Fig. 5B) (treatment $\times$ time interaction, $\left.F_{(69,552)}=2.27 ; p<0.001\right)$ and hence their cocaine intake; rats given $0.1 \mathrm{~mm}$ Kyn $1 \mathrm{~h}$ into their session continued to respond normally (Fig. 5B). Although mean response rate was initially elevated, responding was variable in rats infused with $5.0 \mathrm{~mm}$ Kyn in this condition. Three rats stopped responding after only a few rapid responses, whereas three rats showed classic "extinction bursts" of accelerated responding be- 

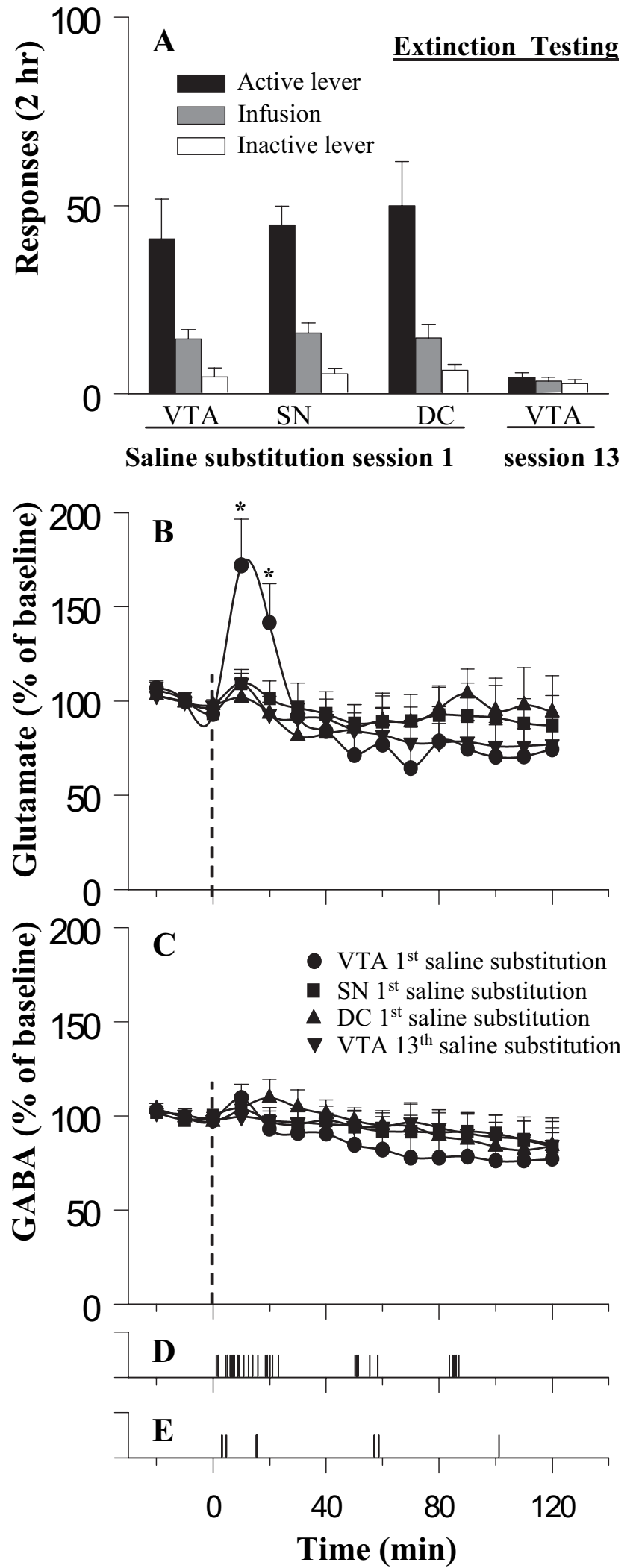

Figure 2. $\quad \boldsymbol{A}-\boldsymbol{C}$, Behavioral responses $(\boldsymbol{A})$ and fluctuations in mean levels of glutamate $(\boldsymbol{B})$ and $\mathrm{GABA}(\boldsymbol{C})$ during extinction. Rats tested for the first time in extinction responded vigorously in the early minutes of the session but did not maintain responding thereafter; a typical response record is shown in $\boldsymbol{D}$. $\boldsymbol{E}$, Rats tested for the 13th time in extinction responded minimally. $B$, Glutamate levels in the VTA but not in the SN or DC groups were significantly increased during the early period of the first substitution session. No such increase was seen on the 13th extinction trial. The dotted vertical lines at time $=0$ indicate the start of the self-administration session. The asterisk $\left(^{*}\right)$ indicates significant $(p<0.05)$ difference from baseline levels.
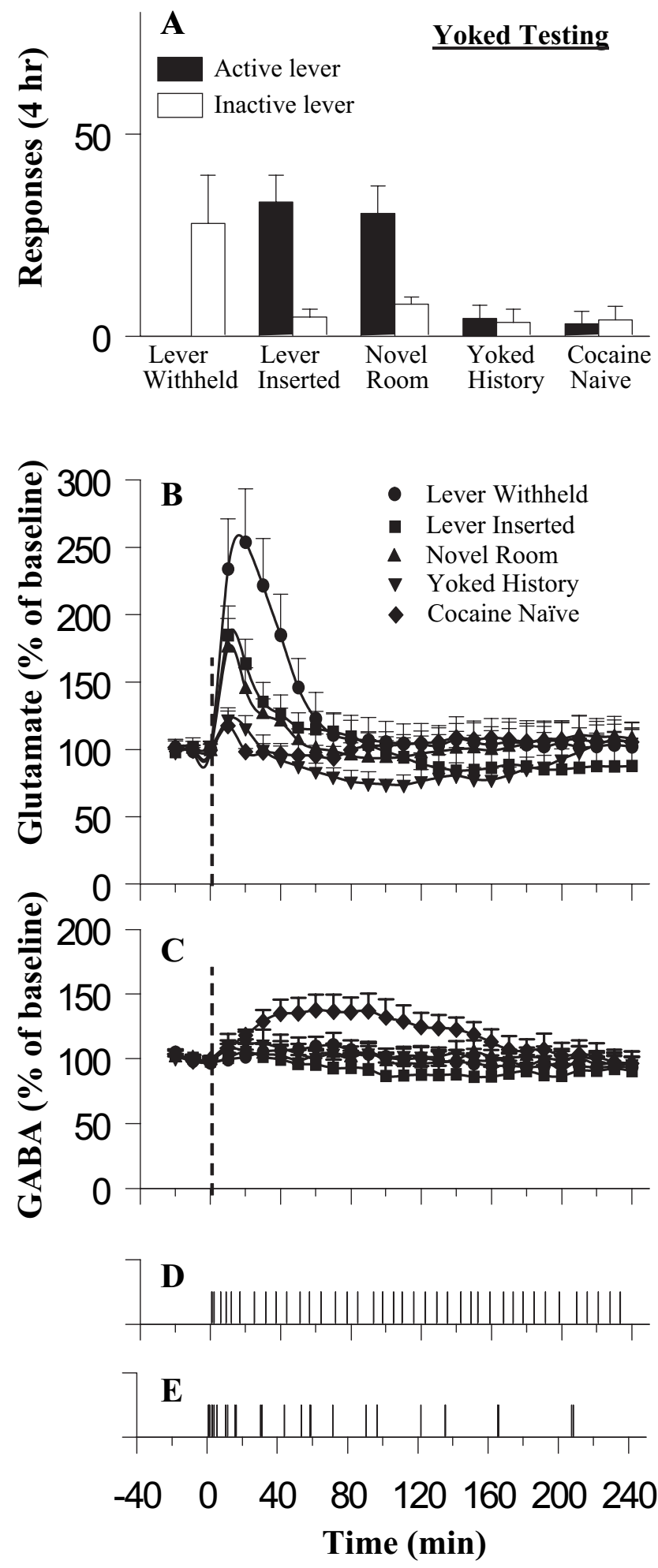

Figure 3. $\quad \boldsymbol{A}-\boldsymbol{C}$, Behavioral responses $(\boldsymbol{A})$ and fluctuations in mean levels of glutamate $(\boldsymbol{B})$ and $G A B A(C)$ in various groups during yoked cocaine infusions. Significant increases in glutamate levels were seen in all groups of cocaine self-administration-trained rats. In order of responsiveness, the groups wereas follows: cocaine-trained animals given yoked infusions in the absence of the lever they would normally press for the drug (lever withheld); cocaine-trained animals given yoked infusions with the lever inserted (lever inserted); animals trained similarly but in a different room and test chamber (novel room); a group that had 2 weeks of experience with yoked infusions before the day of testing (yoked history); a group of saline-trained animals given yoked cocaine infusions for the first time (cocaine naive). $\boldsymbol{D}, \boldsymbol{E}$, The active lever-press pattern of a typical executive animal and a rat from the lever-inserted group, respectively. The dotted vertical lines at time $=0$ indicate the start of the self-administration session. 

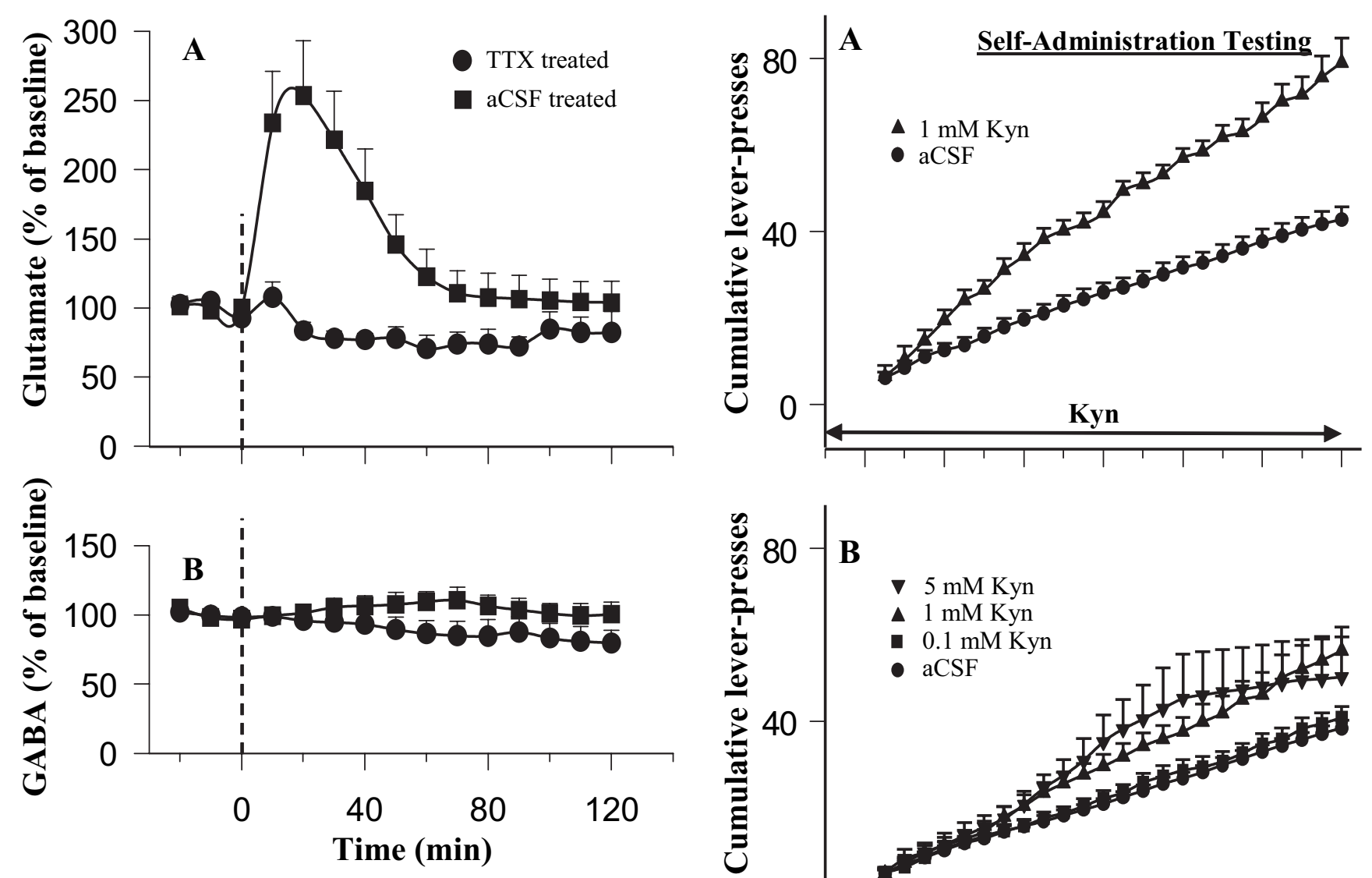

Figure 4. $\quad \boldsymbol{A}, \boldsymbol{B}$, Fluctuations in mean VTA levels of glutamate $(\boldsymbol{A})$ and GABA $(\boldsymbol{B})$ in animals receiving yoked infusions (lever-withheld condition) in the presence (circles) or absence (squares) of TTX perfusion through the dialysis probe. TTX $(0.1 \mu \mathrm{M})$ perfusion began $30 \mathrm{~min}$ before baseline sample collection and continued throughout the experiment. The control group data were from Figure 3 . The vertical doted lines indicate the start of the yoked cocaine infusion testing.

fore ceasing to respond (Fig. 5C). Kyn did not render the animals incapable of normal responding; all animals tested with $1 \mathrm{~mm}$ Kyn responded consistently for the full session at higher than normal rates (Fig. $5 B$ ), and all animals tested with $5 \mathrm{~mm}$ showed higher than normal rates of responding for at least a significant period of the session.
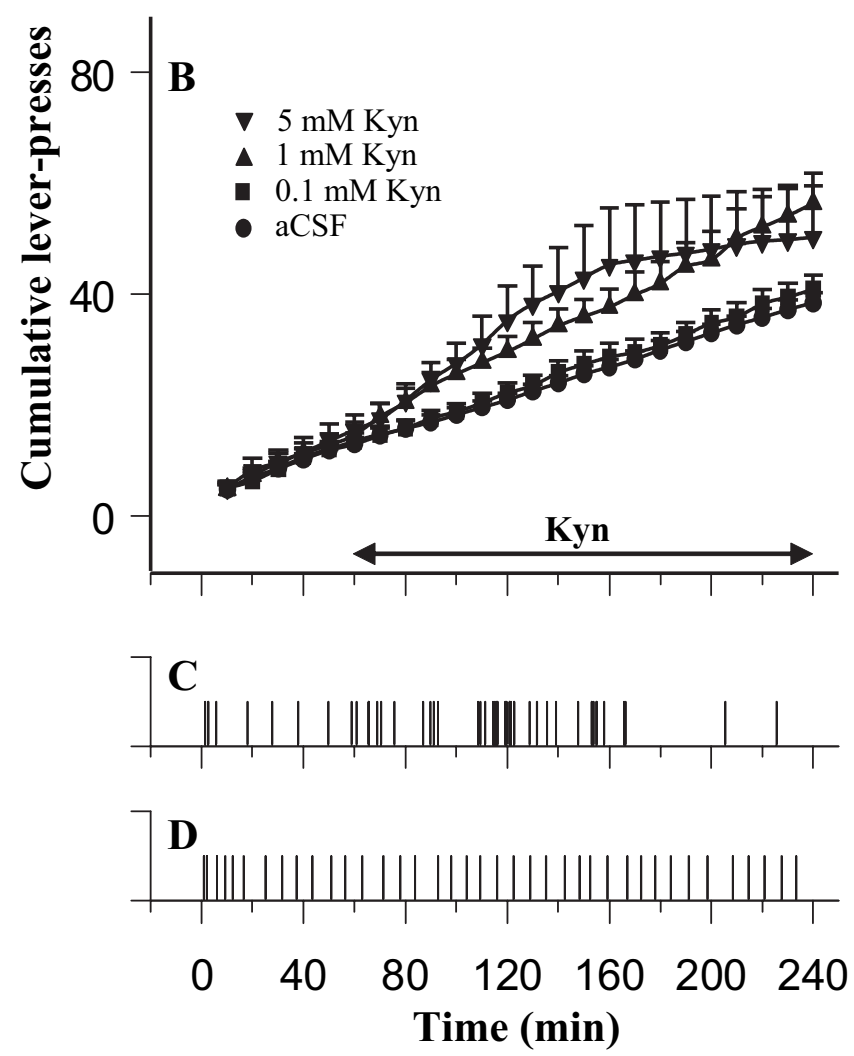

Effects of VTA glutamate receptor blockade on responding in extinction

When Kyn was infused through the VTA dialysis probe during saline substitution (extinction conditions), $1.0 \mathrm{~mm}$ Kyn blocked responding for saline almost immediately and completely (Fig. $6 A$ ) and was almost equally effective at an order of magnitude lower concentration $(0.1 \mathrm{~mm})$ than was ineffective against cocaine self-administration itself (treatment, $F_{(2,18)}=12.44 ; p<$ 0.001 ; time, $F_{(14,252)}=15.3 ; p<0.001$; interaction, $F_{(28,252)}=$ $7.02 ; p<0.001)$. Kyn $(0.1 \mathrm{~mm})$ infusion through $\mathrm{SN}$ or DC cannulas had no significant effect (Fig. 6B). Infusion of AP-5, CNQX, or the combination of AP-5 with CNQX each significantly suppressed responding in extinction (Fig. 6C) (treatment, $F_{(3,24)}=9.94 ; p<0.001$; time, $F_{(14,336)}=19.3 ; p<0.001$; interaction, $\left.F_{(28,252)}=3.53 ; p<0.001\right)$.

Histological examination of the microdialysis membrane locations

Probe placements are shown in Figure 7. The probe membranes of rats with unilateral or bilateral VTA probes were primarily in

Figure 5. Mean response rate during cocaine self-administration as a function of bilatera VTA perfusion of the ionotropic glutamate receptor antagonist Kyn or its vehicle (aCSF). $\boldsymbol{A}, \boldsymbol{B}$, Kyn was perfused $1 \mathrm{~h}$ before $(\boldsymbol{A})$ or $1 \mathrm{~h}$ after $(\boldsymbol{B})$ the beginning of the cocaine self-administration sessions. In $\boldsymbol{A}$, three animals did not initiate responding normally at the beginning of the session; rather, they initiated 12,14 , and 23 min into the session. In $\boldsymbol{B}$, three rats with $5 \mathrm{~mm}$ Kyn perfusion essentially quit responding after $\sim 1 \mathrm{~h}$ of accelerated lever pressing after onset of Kyn infusion. $\boldsymbol{C}, \boldsymbol{D}$, The active lever-press pattern of a typical rat from aCSF perfusion group and one from 5 mм Kyn group, respectively.

the posterior VTA, usually penetrating the parabrachial pigmented and paranigral nuclei, and occasionally partially intruding the rostral interpeduncular nucleus. The membranes of DC rats localized mainly to the magnocellular subregion of the red nucleus. The membranes of SN probes spanned both the zona compacta and the zona reticulata of the SN. One rat in the novelroom group was excluded because of dorsal probe placements. One rat with bilateral VTA probes (used for testing of $1 \mathrm{~mm}$ Kyn on cocaine self-administration) was excluded because of the dor- 

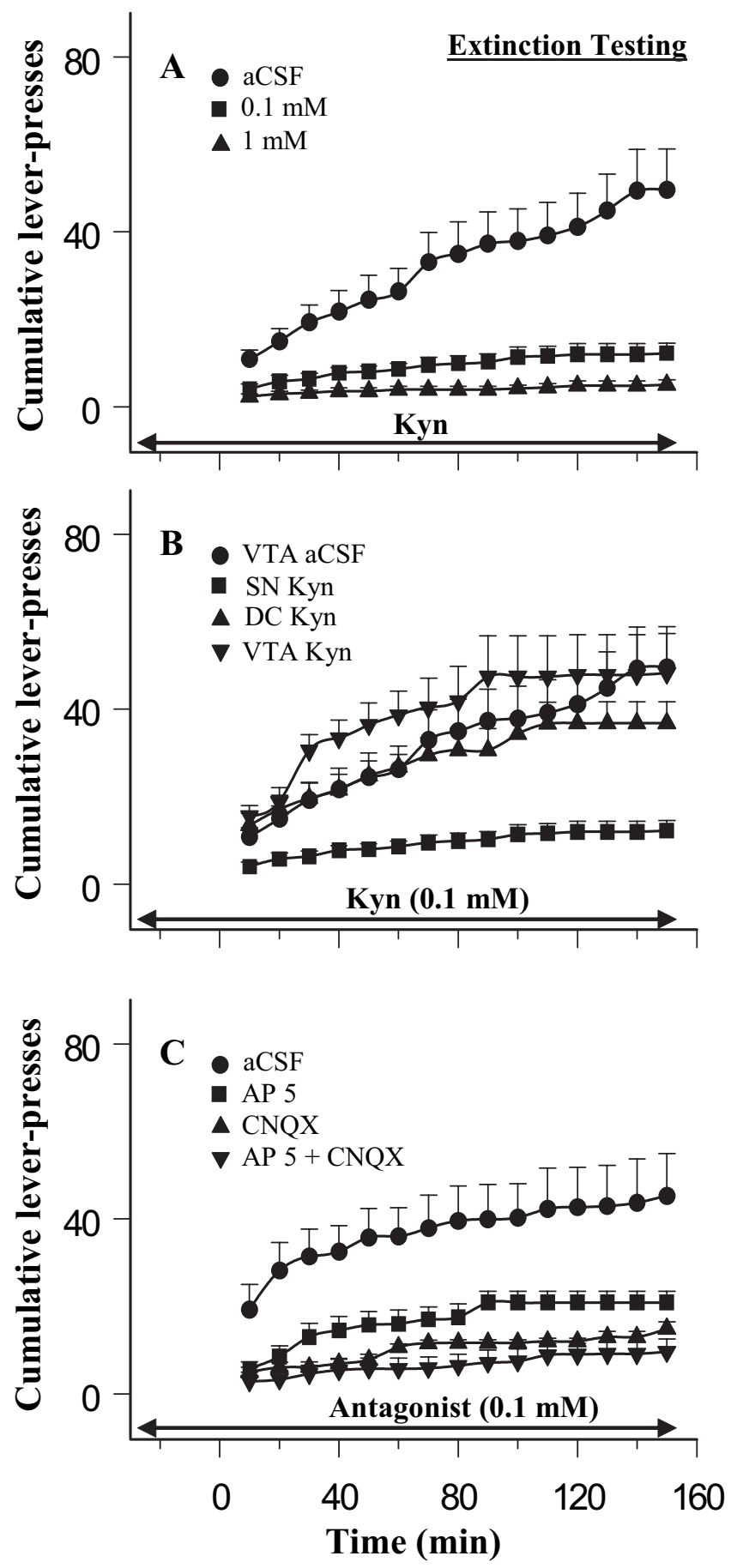

Figure 6. Mean response rate during extinction as a function of bilateral perfusions of various concentrations of the ionotropic glutamate receptor antagonists. Perfusions of antagonists were started $1 \mathrm{~h}$ before the beginning of the extinction testing and continued until the end of the experiment. $\boldsymbol{A}$, The dose comparisons for the nonselective antagonist Kyn. $\boldsymbol{B}$, The site comparisons at $0.1 \mathrm{~mm}$ Kyn perfusion. $C$, The effects of the NMDA-type selective antagonist AP-5 and the AMPA/kainate-type selective antagonist CNQX.

sal placement of one probe. One rat with an intended VTA placement was found to have its probe dorsal to the VTA; its data were included in DC group. Microdialysis samples from one rat in the lever-withheld group were lost because of an HPLC problem.

\section{Discussion}

These findings confirm the discovery of Kalivas and Duffy (1998) that cocaine causes VTA glutamate release only in cocaine- experienced, not cocaine-naive, animals. However, the present findings establish that the glutamate response results from an associative process and is not, as first suspected, a simple consequence of passive pharmacological sensitization. First, cocaine experience was not a sufficient condition for cocaine to cause glutamate release. There was no VTA glutamate response when animals had previously received cocaine only in the form of yoked injections (Fig. 3, Yoked history). The levels of cocaine given to these animals in the training phase were more than sufficient to provide robust and long-lasting psychomotor sensitization (Hooks et al., 1994), yet the animals showed no glutamate response to the test injection.

Moreover, in the present study, similar glutamate responses were seen whether trained animals earned the cocaine they expected (Fig. 1) or earned unexpected saline injections (Fig. 2) in their place. Thus the glutamate responses were not determined by cocaine itself, but rather were determined by the conditions of testing and of the reinforcement history of the animals. This is confirmed by the finding that the glutamate response seen at the time of the first saline substitution was no longer seen at the time of the 13th saline substitution, 2 weeks later. Extinction trials of this type spare the pharmacological sensitization induced by repeated amphetamine (Stewart and Vezina, 1991) but failed to spare the glutamate response. Finally, the fact that the glutamate response is not tied to brain levels of cocaine itself is further indicated by the fact that the glutamate responses abated in $1 \mathrm{~h}$, whereas cocaine intake remained constant for the full $4 \mathrm{~h}$ of the session.

The glutamate responses were sometimes correlated and sometimes not correlated with the initiation or execution of lever pressing. Although glutamate levels and response rate were correlated in animals tested in extinction conditions (first saline substitution) (Fig. 1), they were not correlated in animals that actually received cocaine (cocaine self-administration) (Fig. 1), a condition in which response rate is strongly dominated by pharmacological factors (Dougherty and Pickens, 1974; Yokel and Pickens, 1974; Wise et al., 1995b; Norman and Tsibulsky, 2006). Moreover, glutamate levels were not correlated with lever pressing in the animals with the strongest glutamate response, animals that were not given access to the active lever (lever withheld) (Fig. 2 ); these animals turned to the inactive lever, but inactive lever pressing was not correlated with glutamate elevations. The glutamate responses may, however, have been associated with some aspect of behavioral arousal. The group with the strongest glutamate response was the most obviously activated group; their activity was initially directed at the slot in which the response lever normally appears. The general activity of the other groups that were not under the pharmacological control of cocaine itself was reflected in active lever responding that was, in these groups, correlated with the glutamate elevations.

From the present studies, it cannot be determined whether the glutamate release was a cause or a correlate of cocaine-seeking behaviors. However, the behaviors and the glutamate elevations were each responses to some subset of the stimuli that normally predicted cocaine reward or cocaine availability. These stimuli included lever insertion, cue-light illumination, contextual cues, and perhaps even time of day; for the lever-withheld animals receiving yoked injections, the cues included the drug stimulus itself (cardiac or blood pressure effects of cocaine, pressure, or temperature of the i.v. injections). The cocaine-seeking behaviors included approach to the slot in which the lever normally appeared (the lever pressing itself is only part of the conditioned response habit) and even elevated responding on the inactive 
lever, a generalization from the conditioned response. Neither lever pressing nor glutamate elevations were seen except in animals previously trained to selfadminister cocaine; thus the glutamate responses as well as the lever pressing depended crucially on the cocaine reinforcement history of the animals. Moreover, the expectation of cocaine was sufficient to cause glutamate responses on the first day of extinction training, when cocaine was not actually given. Thus the behavior and the glutamate elevations were each consequences of cocainepredictive stimuli that initiated the onset of cocaine-seeking behaviors. In studies of food reward in monkeys, it has been shown that ventral tegmental and substantia nigra neurons that respond to the touch of a food morsel or the taste of juice in the mouth come to respond to the click of a conditioned stimulus that regularly precedes the touch or the taste (Ljungberg et

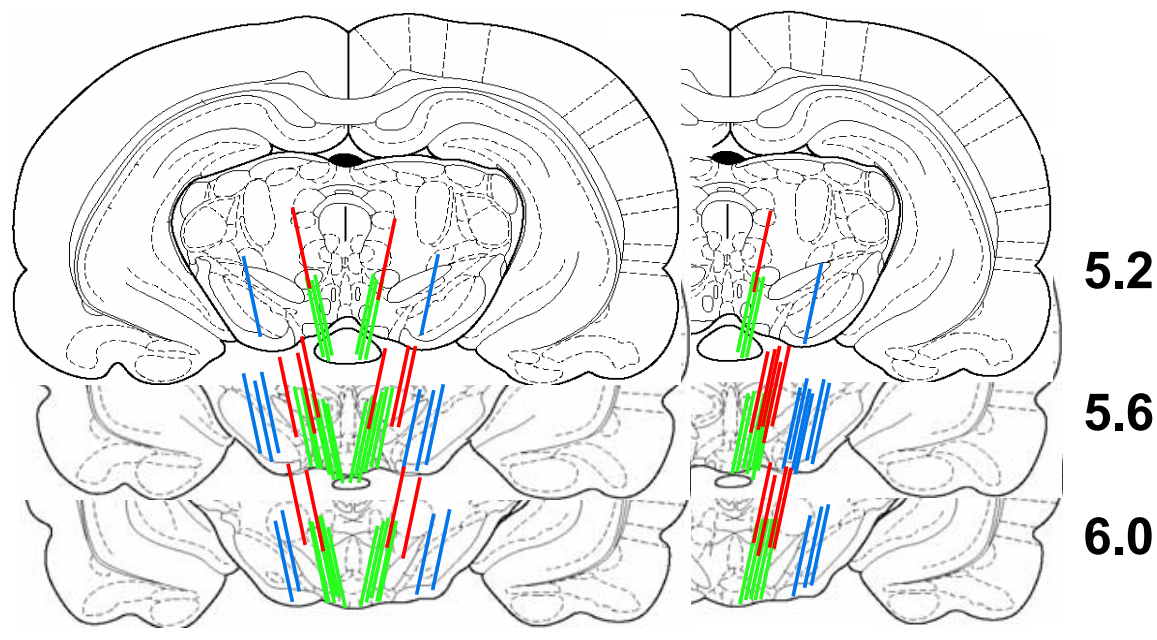

Figure 7. Probe placements. Colored lines indicate the location of the active portion of the dialysis membrane. Right, Unilateral placements for microdialysis collection. Left, Bilateral placements for reverse dialysis experiments. Green, VTA placements; blue, SN placements; red, DC placements. The number of lines for the VTA group is less than the number of animals because of overlap in the placements. Drawings were adapted from the atlas of Paxinos and Watson (1998). The numbers on the right side of the drawings indicate the distance (in millimeters) posterior to the bregma.

al., 1992; Schultz et al., 1993). To the de-

gree that extracellular glutamate in the VTA is an excitatory signal for dopaminergic neuronal activation, glutamate seems a likely candidate for the input that signals such conditioned reward predictors.

We have not yet identified the anatomical source of the glutamate release. The release is blocked by the sodium channel blocker TTX and thus is assumed to reflect transmitter release from glutamatergic afferents to the VTA. The VTA receives glutamatergic input from many sources, including the prefrontal cortex, the lateral hypothalamus, and the dorsomedial and pedunculopontine nuclei (Geisler et al., 2005), and it contains glutamatergic neurons of its own that may synapse on its dopaminergic or GABAergic neighbors (Yamaguchi et al., 2006). VTA dopamine neurons are phasically activated by reward-predicting stimuli (Schultz et al., 1993; Phillips et al., 2003), and the present experiments suggest glutamatergic afferents as one source of such activation. The glutamate peaks measured by microdialysis, however, are much slower than the dopaminergic activations reflected in single-cell recordings or fast-scan voltammetric measurements. This should not be surprising, because of the limited diffusion of synaptic glutamate to the extracellular fluid compartment sampled by microdialysis (Huang and Bergles, 2004). Thus it should be assumed that the time course of the VTA glutamate peaks reported here poorly reflect the timing of the actual glutamate release in the synapse.

The contribution of conditioned VTA glutamate release appears to be attributable to a local glutamate action in the VTA, because conditioned glutamate release was not detected in dialysates either lateral to the VTA in the SN or dorsal to the VTA in the DC condition. Furthermore, glutamate antagonists perfused into the $\mathrm{SN}$ or the DC probes were ineffective against responding in the extinction condition. Although recent evidence suggests differences in the motivational effects of manipulations of the rostral and caudal VTA (Ikemoto and Wise, 2004), such comparisons were not made in the present study.

It seems clear that the conditioned glutamate responses to cocaine-predictive stimuli contribute to the reinforcing effects of cocaine and account for the sustained lever pressing in extinction when cocaine is unexpectedly not given, because the nonselective ionotropic glutamate antagonist Kyn caused animals to behave as if the rewarding effects of the standard cocaine dose had been reduced, and Kyn, the NMDA-type antagonist AP-5, and the AMPA/kainate-type antagonist CNQX each almost completely eliminated responding in extinction. In this case, the most important cue would likely be the cue light that was illuminated with each cocaine infusion during training and with each lever press throughout the $2 \mathrm{~h}$ period of extinction testing. Such stimuli are known to prolong extinction and to serve, for a time, as rewards in their own right (Davis and Smith, 1976). The obvious possibility is that conditioned VTA glutamate release adds to the dopaminergic elevation in nucleus accumbens septi, prolonging the rewarding consequence of each earned cocaine injection during cocaine self-administration and substituting, partially, for cocaine during initial extinction trials.

\section{References}

Carlezon Jr WA, Wise RA (1996) Rewarding actions of phencyclidine and related drugs in nucleus accumbens shell and frontal cortex. J Neurosci 16:3112-3122.

Davis WM, Smith SG (1976) Role of conditioned reinforcers in the initiation, maintenance and extinction of drug-seeking behavior. Pavlov J Biol Sci 11:222-236.

de Wit H, Wise RA (1977) Blockade of cocaine reinforcement in rats with the dopamine receptor blocker pimozide, but not with the noradrenergic blockers phentolamine or phenoxybenzamine. Can J Psychol 31:195-203.

Dommett E, Coizet V, Blaha CD, Martindale J, Lefebvre V, Walton N, Mayhew JE, Overton PG, Redgrave P (2005) How visual stimuli activate dopaminergic neurons at short latency. Science 307:1476-1479.

Dougherty JD, Pickens R (1974) Effects of phenobarbital and SKF 525A on cocaine self-administration in rats. Drug Addict 3:135-143.

Fouriezos G, Wise RA (1976) Pimozide-induced extinction of intracranial self-stimulation: response patterns rule out motor or performance deficits. Brain Res 103:377-380.

Geisler S, Derst C, Gautam M, Veh RW, Zahm DS (2005) Glutamatergic afferents of the ventral tegmental area in rat. Soc Neurosci Abstr 31:517.511.

Goeders NE, Smith JE (1983) Cortical dopaminergic involvement in cocaine reinforcement. Science 221:773-775

Grace AA, Bunney BS (1979) Paradoxical GABA excitation of nigral dopaminergic cells: indirect mediation through reticulata inhibitory neurons. Eur J Pharmacol 59:211-218.

Harris GC, Aston-Jones G (2003) Critical role for ventral tegmental gluta- 
mate in preference for a cocaine-conditioned environment. Neuropsychopharmacology 28:73-76.

Heikkila RE, Orlansky H, Cohen G (1975) Studies on the distinction between uptake inhibition and release of $\left({ }^{3} \mathrm{H}\right)$ dopamine in rat brain tissue slices. Biochem Pharmacol 24:847-852.

Hernandez L, Hoebel BG (1988) Food reward and cocaine increase extracellular dopamine in the nucleus accumbens as measured by microdialysis. Life Sci 42:1705-1712.

Hilmas C, Pereira EF, Alkondon M, Rassoulpour A, Schwarcz R, Albuquerque EX (2001) The brain metabolite kynurenic acid inhibits $\alpha 7$ nicotinic receptor activity and increases non- $\alpha 7$ nicotinic receptor expression: physiopathological implications. J Neurosci 21:7463-7473.

Hooks MS, Duffy P, Striplin C, Kalivas PW (1994) Behavioral and neurochemical sensitization following cocaine self-administration. Psychopharmacology (Berl) 115:265-272.

Huang YH, Bergles DE (2004) Glutamate transporters bring competition to the synapse. Curr Opin Neurobiol 14:346-352.

Hurd JL, Weiss F, Koob GF, Anden N-E, Ungerstedt U (1989) Cocaine reinforcement and extracellular dopamine overflow in rat nucleus accumbens: an in vivo microdialysis study. Brain Res 498:199-203.

Ikemoto S (2003) Involvement of the olfactory tubercle in cocaine reward: intracranial self-administration studies. J Neurosci 23:9305-9511.

Ikemoto S, Wise RA (2004) Mapping of chemical trigger zones for reward. Neuropharmacology 47 [Suppl 1]:190-201.

Jones IW, Wonnacott S (2004) Precise localization of $\alpha 7$ nicotinic acetylcholine receptors on glutamatergic axon terminals in the rat ventral tegmental area. J Neurosci 24:11244-11252.

Kalivas PW, Duffy P (1998) Repeated cocaine administration alters extracellular glutamate in the ventral tegmental area. J Neurochem 70:1497-1502.

Ljungberg T, Apicella P, Schultz W (1992) Responses of monkey dopamine neurons during learning of behavioral reactions. J Neurophysiol 67:145-163.

Morón JA, Brockington A, Wise RA, Rocha BA, Hope BT (2002) Dopamine uptake through the norepinephrine transporter in brain regions with low levels of the dopamine transporter: evidence from knock-out mouse lines. J Neurosci 22:389-395.

Norman AB, Tsibulsky VL (2006) The compulsion zone: a pharmacological theory of acquired cocaine self-administration. Brain Res 1116:143-152.

Paxinos G, Watson C (1998) The rat brain in stereotaxic coordinates, Ed 4. San Diego: Academic.

Pettit HO, Justice JB (1989) Dopamine in the nucleus accumbens during cocaine self-administration as studied by in vivo microdialysis. Pharmacol Biochem Behav 34:899-904.

Phillips PE, Stuber GD, Heien ML, Wightman RM, Carelli RM (2003) Sub- second dopamine release promotes cocaine seeking. Nature 422:614-618.

Ranaldi R, Pocock D, Zereik R, Wise RA (1999) Dopamine fluctuations in the nucleus accumbens during maintenance, extinction, and reinstatement of intravenous D-amphetamine self-administration. J Neurosci 19:4102-4109.

Roberts DCS, Corcoran ME, Fibiger HC (1977) On the role of ascending catecholaminergic systems in intravenous self-administration of cocaine. Pharmacol Biochem Behav 6:615-620.

Schultz W (1997) Dopamine neurons and their role in reward mechanisms. Curr Opin Neurobiol 7:191-197.

Schultz W, Apicella P, Ljungberg T (1993) Responses of monkey dopamine neurons to reward and conditioned stimuli during successive steps of learning a delayed response task. J Neurosci 13:900-913.

Stewart J, Vezina P (1991) Extinction procedures abolish conditioned stimulus control but spare sensitized responding to amphetamine. Behav Pharmacol 2:65-71.

Sun W-L, Akins CK, Mattingly AE, Rebec GV (2005) Ionotropic glutamate receptors in the ventral tegmental area regulate cocaine-seeking behavior in rats. Neuropsychopharmacology 30:2073-2081.

Wang B, Shaham Y, Zitzman D, Azari S, Wise RA, You ZB (2005) Cocaine experience establishes control of midbrain glutamate and dopamine by corticotropin-releasing factor: a role in stress-induced relapse to drug seeking. J Neurosci 25:5389-5396.

Wise RA (2004) Dopamine, learning and motivation. Nat Rev Neurosci 5:483-494.

Wise RA, Schwartz HV (1981) Pimozide attenuates acquisition of lever pressing for food in rats. Pharmacol Biochem Behav 15:655-656.

Wise RA, Spindler J, deWit H, Gerber GJ (1978) Neuroleptic-induced "anhedonia" in rats: pimozide blocks reward quality of food. Science 201:262-264.

Wise RA, Newton P, Leeb K, Burnette B, Pocock P, Justice JB (1995a) Fluctuations in nucleus accumbens dopamine concentration during intravenous cocaine self-administration in rats. Psychopharmacology (Berl) 120:10-20

Wise RA, Leone P, Rivest R, Leeb K (1995b) Elevations of nucleus accumbens dopamine and DOPAC levels during intravenous heroin selfadministration. Synapse 21:140-148.

Yamaguchi T, Sheen W, Morales M (2006) Glutamatergic neurons in the rat ventral tegmental area. Soc Neurosci Abstr 32:483.425.

Yokel RA, Pickens R (1974) Drug level of D- and L-amphetamine during intravenous self-administration. Psychopharmacologia 34:255-264.

Yokel RA, Wise RA (1975) Increased lever pressing for amphetamine after pimozide in rats: implications for a dopamine theory of reward. Science 187:547-549. 